\title{
The Emerging Role of Cardiac Magnetic Resonance Imaging in the Evaluation of Patients with HFpEF
}

\author{
Jessica Webb ${ }^{1,2} \cdot$ Lauren Fovargue $^{2} \cdot$ Kristin Tøndel $^{3,4} \cdot$ Bradley Porter $^{1,2} \cdot$ Benjamin Sieniewicz ${ }^{1,2}$. Justin Gould ${ }^{1,2}$. \\ Christopher Aldo Rinaldi ${ }^{1,2} \cdot$ Tevfik Ismail $^{1,2} \cdot$ Amedeo Chiribiri $^{2} \cdot$ Gerald Carr-White $^{1,2}$
}

Published online: 5 February 2018

(C) The Author(s) 2018. This article is an open access publication

\begin{abstract}
Purpose of Review To give an update on the emerging role of cardiac magnetic resonance imaging in the evaluation of patients with heart failure with preserved ejection fraction (HFpEF). This is important as the diagnosis of HFpEF remains challenging and cardiac imaging is pivotal in establishing the function of the heart and whether there is evidence of structural heart disease or diastolic dysfunction. Echocardiography is widely available, although the gold standard in quantifying heart function is cardiac magnetic resonance $(\mathrm{CMR})$ imaging.

Recent Findings This review includes the recently updated 2016 European Society of Cardiology guidelines on diagnosing $\mathrm{HFpEF}$ that define the central role of imaging in identifying patients with HFpEF. Moreover, it includes the pathophysiology in HFpEF, how CMR works, and details current CMR techniques used to assess structural heart disease and diastolic function. Furthermore, it highlights promising research techniques that over the next few years may become more used in identifying these patients.
\end{abstract}

Summary CMR has an emerging role in establishing the diagnosis of HFpEF by measuring the left ventricular ejection fraction (LVEF) and evidence of structural heart disease and diastolic dysfunction.

Keywords Cardiac magnetic resonance $(\mathrm{CMR}) \cdot$ Diastolic dysfunction $\cdot$ Left ventricular hypertrophy $\cdot$ Left atrial enlargement

\section{Introduction}

The diagnosis of heart failure with preserved ejection fraction (HFpEF) remains challenging as clinical symptoms and signs are nonspecific [1]. Cardiac imaging is pivotal as the recently updated European Society of Cardiology (ESC) guidelines

This article is part of the Topical Collection on Clinical Trials

Jessica Webb

jessica.webb@kcl.ac.uk

1 Department of Cardiology, Guy's and St Thomas' NHS Foundation Trust, St Thomas' Hospital, Westminster Bridge Road, London SE1 7EH, UK

2 Division of Imaging Sciences and Biomedical Engineering, King's College London, London SE1 7EH, UK

3 Division for Methods, Data Collection and Methods, Statistics Norway, Oslo, Norway

4 Department of Mathematical Sciences and Technology, Norwegian University of Life Sciences, Ås, Norway defined the diagnostic criteria for $\mathrm{HFpEF}$ as a left ventricular ejection fraction (LVEF) that is preserved with either evidence of diastolic dysfunction or structural heart disease, typical symptoms and signs of heart failure (HF), and raised natriuretic peptides $[2 \bullet \bullet]$. Practically, echocardiography is widely used with up-to-date recommendations for assessment of diastolic function [3], although these are indirect assessments of LV filling and lack characterization of myocardial tissue. Cardiac magnetic resonance (CMR) imaging represents the gold standard in quantification of LVEF [4] and is increasingly used in the assessment of HF due to its unique, precise, noninvasive phenotypic characterization with high reproducibility and sufficient spatial and temporal resolution [5]. The role of CMR in characterizing diastolic function has not been fully established clinically, although it is a class I indication for a CMR in HF patients with poor acoustic windows, partly due to the need for additional sequence acquisitions and timeconsuming image analysis and post-processing. This review evaluates the pathophysiology of HFpEF, how CMR works, current CMR techniques used to assess diastolic function, and highlights promising research techniques. 


\section{Pathophysiology of HFpEF}

The exact pathophysiology of HFpEF remains uncertain and is likely due to diastolic dysfunction, impaired systolic function on exercise, abnormal ventricular-arterial coupling, inflammation and endothelial dysfunction, chronotropic incompetence, altered myocardial energetics and peripheral skeletal muscle metabolism and perfusion, and pulmonary hypertension and renal insufficiency [6••]. Only recently has it been convincingly demonstrated that HFpEF represents more than a sum of all its comorbidities and is a condition in its own right [7]. Despite variations in pathophysiology, impaired diastolic ventricular filling is consistently reported across all patients, caused by increased stiffness of the left ventricle, impaired atrial-ventricular conduction of blood, and decreased relaxation capability of the myocytes $[6 \bullet \bullet, 8]$.

\section{What Is Diastolic Dysfunction?}

Diastole is the time taken for the left ventricle to fill between the closure of the aortic valve and the closure of the mitral valve. During this time, blood flows from the atria to the ventricles due to the pressure difference between the chambers with atrial contraction contributing to the final part of ventricular filling. Diastole is an active, ATP-consuming myocardial process. LV filling and the transmitral pressure gradient are determined by the LV and atrial geometry, loading conditions, viscoelastic properties, pericardial restraint, valve function, heart rate, and temporal synchronization of the atrial and ventricular contraction.

Diastolic dysfunction is classified into four phases [9], Fig. 1. In grade 1 diastolic dysfunction, the proportion of LV filling occurring in the early phase of diastole is reduced. Pressure in the left atrium increases as the diastolic function reduces further (grade 2). Grade 3 dysfunction occurs when there is restrictive filling due to impaired relaxation with elevated filling pressure and impaired LV compliance. With the Valsalva maneuver, the impaired filling can be reversed. Grade 4 diastolic dysfunction represents irreversible restrictive filling.

\section{How CMR Works}

CMR uses a magnetic field, approximately 30,000 times stronger than the earth's magnetic field, to align the nuclear magnetization of hydrogen atoms that are abundant in the human body. Image contrast is generated by the hydrogen nuclei being intermittently excited by radiofrequency pulses, resulting in longitudinal and transverse relaxation times that are characteristic for different tissues. The CMR sequence details the instructions for the radiofrequency pulses, timed data acquisitions, and magnetic gradient field switches. Spin echo sequences are mainly used for anatomic imaging and tissue characterization, whereas gradient echo sequences are used to acquire moving images. To prevent artifacts from cardiac and respiratory motion, images are gated to the $\mathrm{R}$ wave of the electrocardiogram and acquired in end-expiratory breath hold. Intravenous gadolinium chelated contrast agents are used to identify areas of scar or fibrosis, as a lengthened washout indicates reduced functional capillary density in the irreversibly injured myocardium.

\section{Current CMR Measurements to Assess Diastolic Dysfunction}

In assessing a patient with HF symptoms, the LVEF first needs to be calculated. The current techniques to assess diastolic dysfunction include measuring left atrial enlargement (LAE), left ventricular hypertrophy (LVH), mitral inflow pattern, pulmonary vein assessment, LV time volume relations, LV myocardial tagging, flow propagation velocity, and calculating T1/ myocardial fibrosis, Fig. 2.

\section{Left Atrial Size}

Left atrial (LA) size, volume, and wall mass can be accurately measured by CMR, and Simpson's volumetric method has always been considered the gold standard for measuring atrial volumes [9]. LA volume can be measured accurately from CMR imaging using the biplane area-length (BAL) technique 2 and 4 chamber images with the following equation: LA volume $(\mathrm{ml})=\left(0.85 \times \mathrm{A}_{2 \mathrm{C}} \times \mathrm{A}_{4 \mathrm{C}}\right) / \mathrm{L}$ (where A2C and $\mathrm{A} 4 \mathrm{C}$ are the $L A$ areas on the 2 chamber and 4 chamber views, and $\mathrm{L}$ is the shorter length of the LA, from either the 2 or 4 chamber). Recent work in patients with HFpEF has shown that the CMR BAL technique is as accurate as CMR volumetric Simpson's method, with echocardiography BAL technique less accurate than CMR volumetric assessment particularly with LA dilatation and in patients with atrial fibrillation [10]. However, the BAL techniques were not compared for echocardiography and CMR. The size of the atrium varies during the cardiac cycle but only maximal LA size is typically reported clinically. Body size is a major determinant of atrial size with little variation noted with gender [11].

The left atrium (LA) has three functions: during ventricular systole, it acts as a reservoir receiving blood from the pulmonary veins and storing energy in the form of pressure; during early diastole, it acts as a conduit for transfer of blood into the left ventricle via a pressure gradient and finally, it has a contractile function, augmenting the LV stroke volume. In patients with normal diastolic function, the relative contribution of the reservoir, conduit, and contractile function of the atria to the LV filling is approximately 40,35 , and $25 \%$, respectively [12]. With abnormal LV relaxation, the relative contribution of atrial reservoir and contractile function increases, and conduit function decreases. The atria enlarge in response to both 


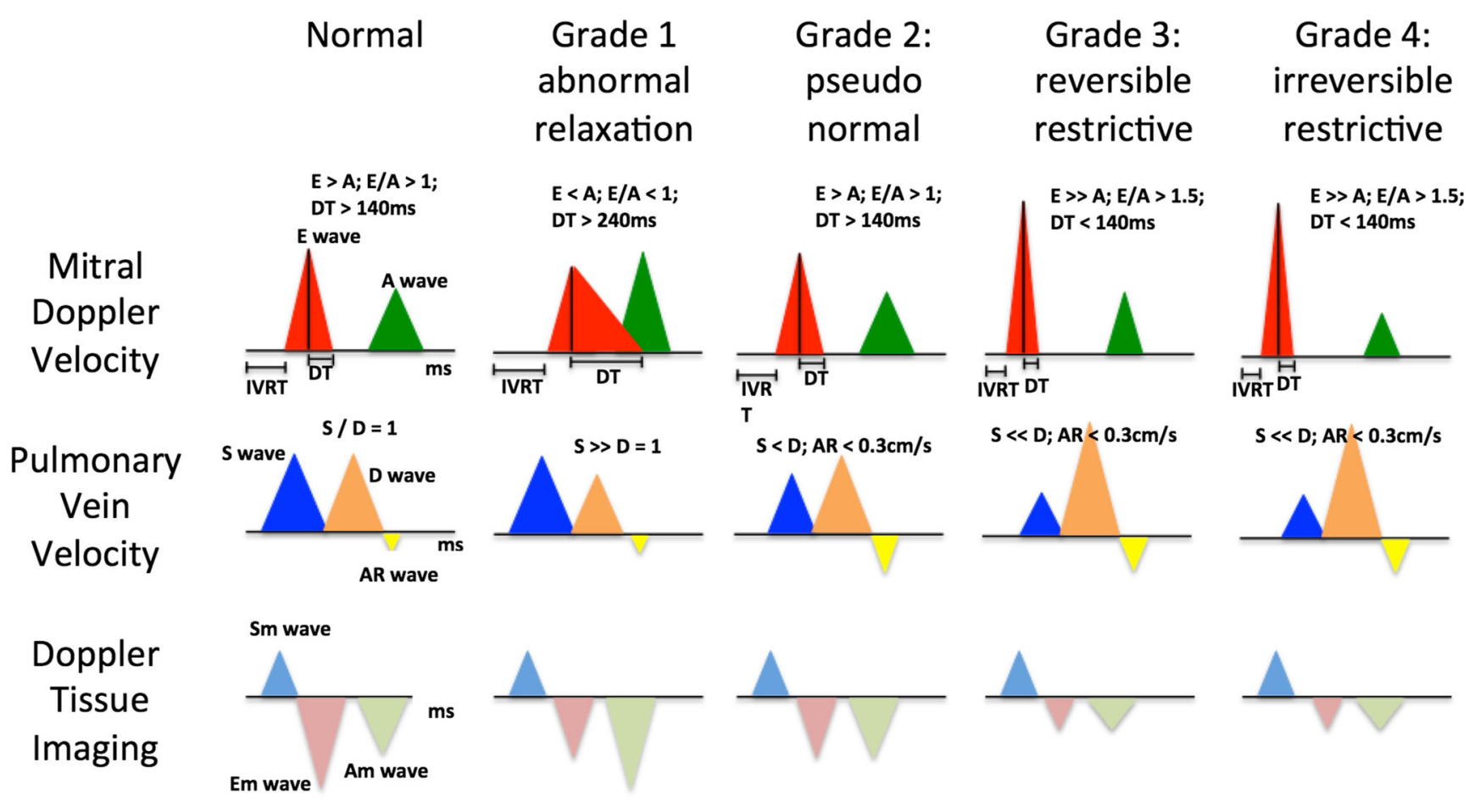

Fig. 1 Classification of diastolic dysfunction

pressure and volume overload. Pressure overload is usually secondary to increased atrial afterload, either mitral valve disease or LV dysfunction, and is uniformly accompanied by abnormal myocyte relaxation. Volume overload, however, caused by athletic hearts, anemia, and mitral regurgitation can cause atrial enlargement and is normally associated with normal ventricular myocardial relaxation.
Atrial size is known to be prognostic; more than $32 \mathrm{ml} / \mathrm{m}^{2}$ is associated with increased incidence of heart failure independent of age, LV hypertrophy, diabetes, hypertension, myocardial infarction of mitral inflow velocities [13]. However there is little evidence on the diagnostic and prognostic value of left atrial volume in HFpEF patients [14], although recent evidence suggests that LA function (measured by ejection
Fig. 2 Anatomy in blue, abnormalities in HFpEF in white

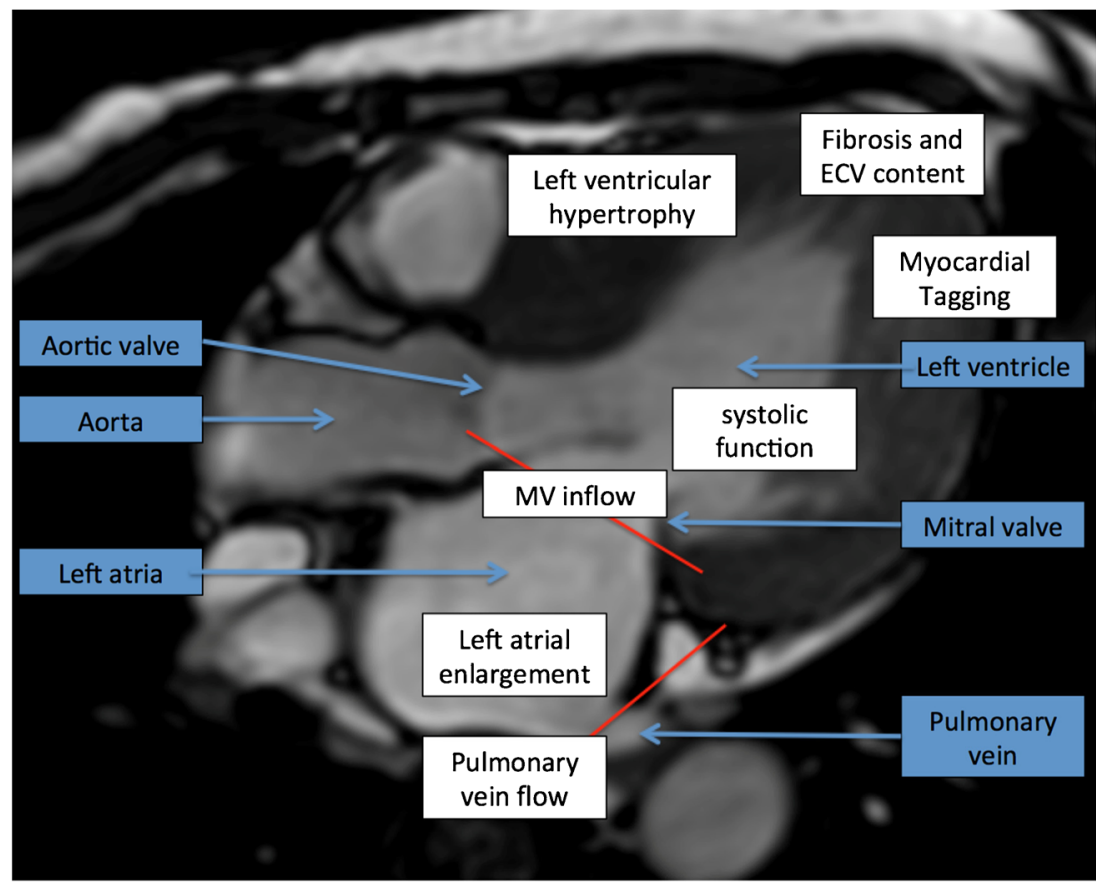


fraction) has been shown to be associated with mortality outcomes [15] and LAE was associated with the presence of dyspnea [16].

\section{Left Ventricular Hypertrophy}

The Framingham Study reported that increased left ventricular (LV) mass is associated with a significant excess of cardiovascular mortality and morbidity, independent of hypertension of the presence of coronary artery disease $[17,18]$. Left ventricular hypertrophy (LVH) is the most common structural abnormality in patients with HFpEF. It is the response of myocytes to various stimuli leading to myocyte hypertrophy, such as increased mechanical load, neurohumoral activation, and cytokines associated with arterial hypertension, diabetes, chronic renal impairment, and other comorbidities [19].

Practically, LVH can be considered as concentric remodeling (enlarged heart with normal relative wall thickness), concentric hypertrophy (increased relative wall thickness with normal LV diameter), or eccentric remodeling (increased relative wall thickness with increased LV diameter). The accuracy of CMR has been validated ex vivo using post-mortem hearts $[20,21]$ and it is more reproducible than both M mode and 2D echocardiography [22].

\section{Mitral Inflow Pattern}

One of the most widely applied techniques in assessing LV diastolic function is the evaluation of the transmitral inflow of pulmonary venous flows using Doppler echocardiography. These results are load-dependent and can change dramatically with only minimal changes in heart rate or preload, and so not evaluate $L V$ relaxation directly.

Despite these limitations, phase contrast MRI is an attractive alternative to echocardiographic pulsed wave Doppler as it allows both quantitative assessment of blood velocity and optimal positioning in 3D space of the tomographic plane of interest ensuring accurate alignment and measurements. The acquisition plane is typically perpendicular to the flow direction at the position of the mitral valve at end systole. Data is retrospectively ECG gated and can be acquired either free breathing or with a breath hold. Thirty to 40 cardiac phases are reconstructed resulting in a typical effective temporal resolution of the time velocity curve of $20-30 \mathrm{~ms}$.

A time-resolved acquisition with velocity encoding perpendicular to this plane results in a time velocity curve representing one average cardiac cycle. The waveform analysis results in quantification of the early (E) and atrial (A) peak filling velocities, E/A ratio, and deceleration time of the $\mathrm{E}$ peak filling velocity. These can be used to classify different grades of diastolic function $[9,23]$.

When compared to echocardiographic results, the results of phase contrast CMR results correlated $100 \%$, although E and
A velocities were found to be systematically lower. However, the CMR acquisition of velocities in a pulsatile flow phantom correlated correctly. These differences in absolute values reflect differences in the nature of acquisition and did not result in any misclassification of diastolic flow abnormalities between modalities [24]; echo data is collected over one cardiac cycle, whereas CMR data is effectively averaged overall several cycles. These acquisitions take between $20 \mathrm{~s}$ and $3 \mathrm{~min}$.

One challenge in CMR, however, is that the acquisition plane remains fixed during the cardiac cycle and does not move with the cyclical motion of the mitral annulus. Acquisition techniques have been introduced using moving slice velocity mapping [25] and three-dimensional three-directional velocity encoding [26]. These have shown better agreement with echo Doppler when discriminating restrictive filling patterns from other grades of diastolic dysfunction [27].

\section{Pulmonary Vein Assessment}

Phase contrast MRI can also assess pulmonary veins. Rathi et al. found that only $68 \%$ of echo cases were able to assess the pulmonary veins, compared to $100 \%$ with CMR [24]. The blood flow waveforms of the pulmonary veins provide important information on diastolic function as LV filling and compliance, left atrial preload, and contractility all influence the left atrial filling pattern. Waveform analysis of the pulmonary venous time velocity curve includes the peak systolic velocity (S), peak anterograde diastolic velocity (D), and the peak atrial reversal velocity (Ar) in late diastole. Often there are two ventricular systolic velocity peaks, S1 and S2. S1 is related to atrial relaxation and S2 to atrial stroke volume and pulse wave propagation in the pulmonary artery. For this reason, S2 should be used to determine $\mathrm{S} / \mathrm{D}$ ratio as a measure of diastolic function. Just like transmitral Doppler, the sample is placed $0.5 \mathrm{~cm}$ into the right upper pulmonary vein and flow velocity indices are influenced by age, although changes in LV filling and compliance affect $\mathrm{D}$ velocity more. Ar velocity increases with age but this does not usually exceed $35 \mathrm{~cm} / \mathrm{s}$ and so values above this suggest increased $\mathrm{LV}$ end-diastolic pressure [28]. A difference in Ar-A duration > $30 \mathrm{~ms}$ indicates elevated LV end-diastolic pressure [23].

\section{Time Volume Relations}

The LV time-volume relation is recorded from a series of breath-held time-resolved parallel short-axis planes or a radial stack of long-axis planes. Segmenting and summating the endocardial borders results in the end-diastolic and end-systolic LV volumes. The stroke volume and systolic function can be calculated from the difference between the end-diastolic and end-systolic volumes. Early peak filling 
rate, time to peak filling rate, and atrial filling fraction can also be calculated from this information. Diastolic dysfunction is associated with decreased peak filling rates and increased time to peak filling rates. Despite improvements in the definition of endocardial borders and the use of automated segmentation [29], the accuracy remains too low to use in clinical practice [30] and these parameters have limited use.

\section{Myocardial Tagging}

It is possible to label or "tag" myocardium by selective saturation pulses in specific regions in planes perpendicular to the imaging plane, Fig. 3. Tag lines appear as black lines due to the saturation of signal from protons and they last for several hundred milliseconds. These lines can be tracked throughout the cardiac cycle, enabling calculations in longitudinal, radial, and circumferential directions, and are obtained every 20 milliseconds resulting in high temporal resolution. The challenges that have prevented this technique from being introduced widespread clinically are low signal to noise ratio, long acquisition times, limited availability of validated postprocessing software, and that the tag lines fade during the cardiac cycle, with $3 \mathrm{~T}$ better than $1.5 \mathrm{~T}$ due to longer $\mathrm{T} 1$ and so longer persistence of the tag.

Edvardsen et al. demonstrated an association with regional diastolic dysfunction in 218 asymptomatic patients with left ventricular hypertrophy who had no evidence of clinical cardiovascular disease or LV dysfunction [31]. More recent work has been directed at feature tracking, a method of postprocessing routine cine acquisitions to provide quantitative measurements of circumferential and radially directed wall strain. However, inter-study reproducibility has been shown to be poor for segmental and long-axis analyses of strain [32]. Additionally, displacement-encoded stimulated echoes (DENSE) and strain-encoded imaging (SENC) applications have both been used.

\section{Flow Propagation Velocity}

The flow propagation velocity $(\mathrm{Vp})$ is a measure of the $\mathrm{LV}$ suction force that has been attributed to LV relaxation. In addition to diastolic function, variables such as flow field, viscoelastic properties, inertial force, LV geometry, systolic function, mitral valve function, and $\mathrm{LV}$ contractile function influence intraventricular flow. A Vp $>50 \mathrm{~cm} / \mathrm{s}$ in echocardiography is considered to be normal. Attempts with threedimensional three-directional velocity encoding CMR may provide quantitative information on the intraventricular blood flow field, although long acquisition times may limit the universal uptake of this technique [33].

\section{Myocardial Fibrosis and Extracellular Volume Measurements}

Myocardial fibrosis is a pathological increase in the myocardial collagen content caused by increased collagen synthesis in the interstitium (resulting in diffuse myocardial fibrosis) or myocyte replacement (resulting in scarring) [34]. Increased diffuse myocardial fibrosis is a major determinant of altered diastolic filling and systolic pumping function of the LV. As myocardial stiffness increases in line with the development of fibrosis, there is subsequent deterioration in both systolic and diastolic function. The pathophysiology of accumulating collagen content is diverse [34]. In HFpEF patients, there is increased collagen synthesis with elevated serum fibrotic biomarkers [35]. There has been controversy as to whether HFpEF patients have a higher collagen volume fraction (CVF). Borberly et al. looked at endomyocardial biopsies taken from HFpEF patients and demonstrated a higher CVF compared to nonHFpEF patients [36]. Although, Aoli et al. studied $172 \mathrm{HFpEF}$ and HFrEF patients' endomyocardial biopsies and found no statistically significant difference between CVF in the HFpEF and HFrEF population and that CVF did not predict outcome in HFpEF patients [37]. More recently, myocardium measured directly from HFpEF patients has been found to have a significant increase in insoluble and total collagen, as well as CVF when compared to patients with hypertension but no HFpEF [38•].

Diffuse interstitial fibrosis, a precursor for replacement fibrosis, is not detected by late gadolinium enhancement but correlates with $\mathrm{T} 1$ mapping, which allows a quantitative assessment of diffuse cardiac fibrosis and estimations of the extracellular matrix volume (ECV) [39-41]. Studies have validated T1 mapping and ECV estimation by CMR against histology in a variety of conditions including aortic stenosis, hypertrophic cardiomyopathy, and amyloidosis, reporting a good correlation with myocardial collagen content $(r=$ $0.51 \sim 0.81)$ [42].

T1 mapping reflected diffuse fibrosis in 25 heart failure patients (LV ejection fraction $35+/-3.3 \%$ ) [41]. In 2014, Mascherbauer et al. demonstrated T1 times, left atrial size, and pulmonary vascular resistance were significantly associated with cardiac events in 100 suspected HFpEF patients. The authors concluded that post-contrast $\mathrm{T} 1$ times are associated with prognosis and should be considered a possible HFpEF biomarker [43]. Another study looked at 62 patients with HFpEF defined as signs and symptoms of heart failure, LVEF $>45 \%$ and LV diastolic dysfunction documented by tissue Doppler echocardiography (mean septal and lateral mitral annular velocity $<8 \mathrm{~cm} / \mathrm{s}$ ) [44]. The authors found a significantly higher ECV in patients with both HFrEF and $\mathrm{HFpEF}$, compared to patients without heart failure. In 
Fig. 3 a Tag image of the myocardium; $\mathbf{b} 2$ chamber view; $\mathbf{c}$ 4 chamber view; $\mathbf{d}$ short-axis image; e tag output for 17 segments of the LV showing deformation against time
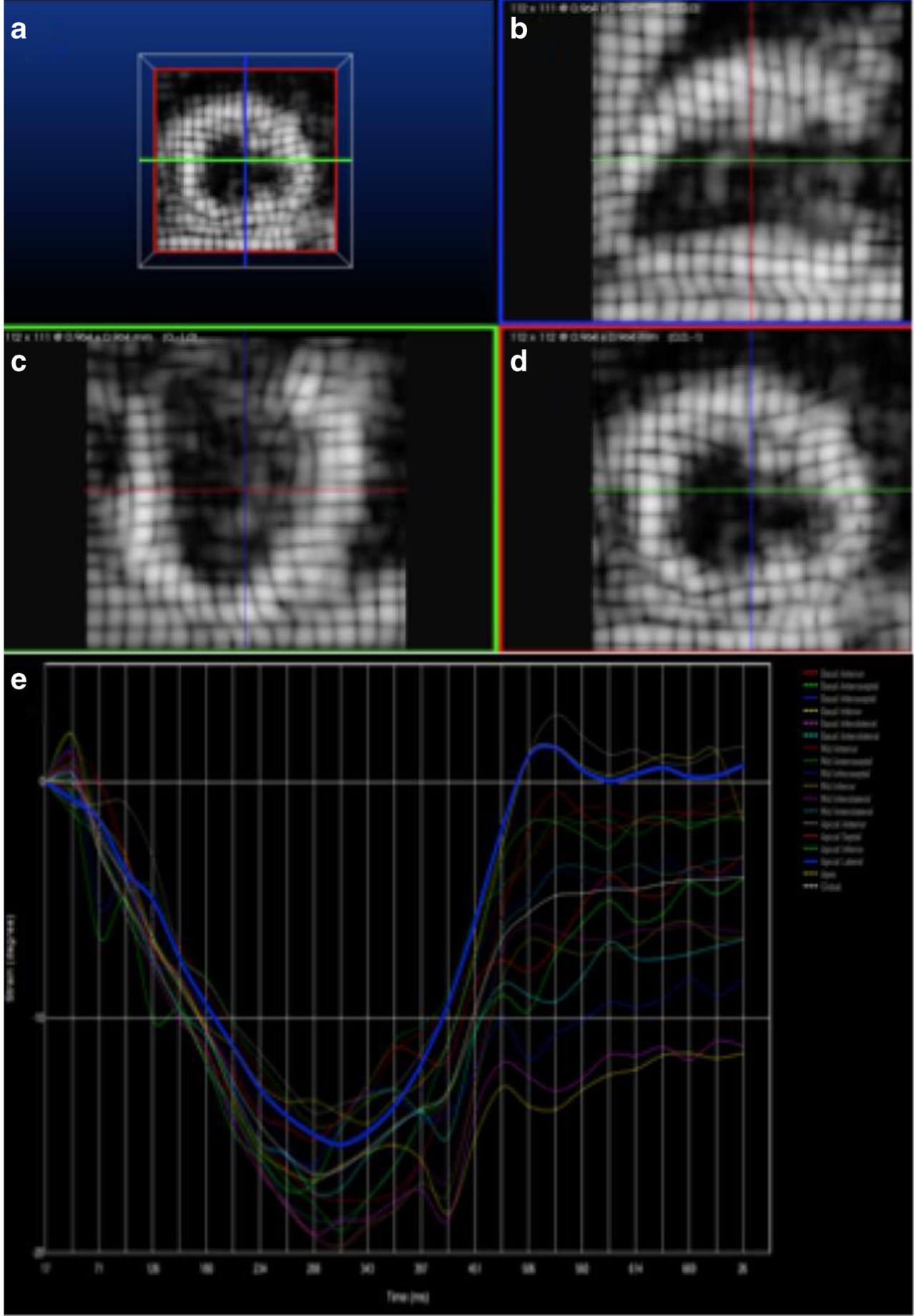

addition, ECV correlated with peak filling rate in the HFpEF population despite no correlation identified in the HFrEF population.

\section{Emerging CMR Imaging Techniques}

Despite recent development in these techniques and the introduction of updated ESC diagnostic guidelines, no one assessment is definitive in detecting patients with HFpEF. Recent research in pulse wave velocity, MR elastography, and 4D flow assessment looks promising in these patients.

\section{ME Elastography}

It has been hypothesized that magnetic resonance elastography (MRE) may act as a biomarker for patients with $\mathrm{HFpEF}$ as it uses mechanical shear waves to quantitatively assess the stiffness of tissues. Applying stress to a material 
results in a strain response of that tissue which can be measured. When the material is subjected to periodic shear, shear waves occur. The pair of equal forces acting in opposite directions along the two faces of the layer causes a change of shape, even though the volume remains unchanged. If the medium is elastic, then the layer will resume its original shape after the shear wave has passed. A wave is propagated as the adjacent layers undergo transient shear with equal forces acting in opposite directions, and then resume their original shape. MR elastography has been shown to map the shear stiffness of soft tissues [45]. In 2017, Arani et al. reported that 16 patients with cardiac amyloid had increased myocardial stiffness in comparison to 11 volunteers (median $11.4 \mathrm{kPa}$ with range of 9.2-15.7, median $8.2 \mathrm{kPa}$ with a range of 7.211.8 , respectively, at a vibration frequency of $140 \mathrm{~Hz}$ ) [46•].

\section{D Flow Assessment}

3D phase contrast MRI, known as 4D CMR, allows volumetric flow imaging in a single acquisition. Complex blood flow patterns can be intuitively visualized as they unfold in multiple dimensions over time. Diastolic dysfunction is associated with abnormal intracardiac blood flow and it is possible to analyze quantitatively with 4D flow. This analysis can identify and monitor diastolic dysfunction. Blood transiting the left ventricle can be divided into four separate functional components: direct flow which enters and exits a single heartbeat, retained inflow which enters but does not exit, delayed ejection flow which exits on the subsequent heartbeat, and the residual volume that resides in the ventricle for at least two cardiac cycles. Investigation of these relative percentages of flow components and the kinetic energy and momentum will allow a refined analysis of the dynamic of ventricular filling and ejection. 4D flow can uniquely identify flow differences not seen with conventional hemodynamic assessment. Diminished direct flow and reduced kinetic energy of the direct flow at end diastole can be identified. As a consequence, increased workload is placed on the left ventricle to eject the same stroke volume.

\section{Limitations of CMR}

There is no doubt that CMR offers excellent spatial resolution and is the gold standard for assessment of LV volumes and myocardial tissue characterization. However, not all departments have access to CMR imaging and the scanners are more expensive that transthoracic echocardiography, as well as taking longer. Furthermore, patient selection is critical as some patients cannot tolerate the scan due to claustrophobia (although there are limited scanners that patients are not enclosed) and patients with certain implants are not allowed in the scanner. There is a small risk of anaphylaxis due to contrast, and patients with renal impairment need reduced doses with contrast administration being contraindicated if the GFR $<15 \mathrm{mls} / \mathrm{min}$.

\section{Conclusion}

Cardiac MRI is fundamental in the assessment and characterization in heart failure patients. Despite challenges with long acquisition times and challenges in post-processing, CMR has a pivotal role in measuring LV systolic function and identifying left atrial enlargement and left ventricular hypertrophy, as well as other measures of diastolic function.

\section{Compliance with Ethical Standards}

Conflict of Interest Jessica Webb, Lauren Fovargue, Kristin Tøndel, Bradley Porter MBBS, Ben Sieniewicz, Justin Gould, Christopher Aldo Rinaldi, Tevfik Ismail, Amedeo Chiribiri and Gerald Carr-White declare no conflicts of interest.

Human and Animal Rights and Informed Consent This article does not contain any studies with human or animal subjects performed by any of the authors.

Open Access This article is distributed under the terms of the Creative Commons Attribution 4.0 International License (http:// creativecommons.org/licenses/by/4.0/), which permits unrestricted use, distribution, and reproduction in any medium, provided you give appropriate credit to the original author(s) and the source, provide a link to the Creative Commons license, and indicate if changes were made.

\section{References}

Papers of particular interest, published recently, have been highlighted as:

- Of importance

•• Of major importance

1. Nikitin NP, Witte KK, Thackray SD, de Silva R, Clark AL, Cleland JG. Longitudinal ventricular function: normal values of atrioventricular annular and myocardial velocities measured with quantitative two-dimensional color Doppler tissue imaging. J Am Soc Echocardiogr. 2003;16(9):906-21. https://doi.org/10.1016/S08947317(03)00279-7.

2.• Ponikowski P, Voors AA, Anker SD, Bueno H, Cleland JG, Coats AJ, et al. 2016 ESC guidelines for the diagnosis and treatment of acute and chronic heart failure: the task force for the diagnosis and treatment of acute and chronic heart failure of the European Society of Cardiology (ESC). Developed with the special contribution of the Heart Failure Association (HFA) of the ESC. European heart journal. 2016. These are the updated ESC guidelines on Heart Failure that define the diagnostic and treatment pathways for each type of $\mathrm{HF}$.

3. Mathew T. A guideline protocol for the echocardiographic assessment of diastolic dysfunction. British Society Echocardiography. 2013;November.

4. Grothues F, Smith GC, Moon JC, Bellenger NG, Collins P, Klein $\mathrm{HU}$, et al. Comparison of interstudy reproducibility of 
cardiovascular magnetic resonance with two-dimensional echocardiography in normal subjects and in patients with heart failure or left ventricular hypertrophy. Am J Cardiol. 2002;90(1):29-34. https://doi.org/10.1016/S0002-9149(02)02381-0.

5. Baksi AJ, Pennell DJ. T1 mapping in heart failure: from technique to prognosis, toward altering outcome. Circ Cardiovasc imaging. 2013;6(6):861-3. https://doi.org/10.1161/CIRCIMAGING.113. 001178.

6.• Sharma K, Kass DA. Heart failure with preserved ejection fraction: mechanisms, clinical features, and therapies. Circ Res. 2014;115(1):79-96. https://doi.org/10.1161/CIRCRESAHA.115. 302922. This is an excellent review paper with perspectives on its evolving demographics and epidemiology as well as potential mechanisms involving the heart and other organs

7. Mohammed SF, Borlaug BA, Roger VL, Mirzoyev SA, Rodeheffer RJ, Chirinos JA, et al. Comorbidity and ventricular and vascular structure and function in heart failure with preserved ejection fraction: a community-based study. Circ Heart Fail. 2012;5(6):710-9. https://doi.org/10.1161/CIRCHEARTFAILURE.112.968594.

8. Zile MR, Baicu CF, Gaasch WH. Diastolic heart failure - abnormalities in active relaxation and passive stiffness of the left ventricle. N Engl J Med. 2004;350(19):1953-9. https://doi.org/10.1056/ NEJMoa032566.

9. Paulus WJ, Tschope C, Sanderson JE, Rusconi C, Flachskampf FA, Rademakers FE, et al. How to diagnose diastolic heart failure: a consensus statement on the diagnosis of heart failure with normal left ventricular ejection fraction by the Heart Failure and Echocardiography Associations of the European Society of Cardiology. Eur Heart J. 2007;28(20):2539-50. https://doi.org/10. 1093/eurheartj/ehm037.

10. Vassiliou VS, Patel HC, Rosen SD, Auger D, Hayward C, Alpendurada $\mathrm{F}$, et al. Left atrial dilation in patients with heart failure and preserved ejection fraction: insights from cardiovascular magnetic resonance. Int J Cardiol. 2016;210:158-60. https://doi.org/10. 1016/j.ijcard.2016.02.101.

11. Abhayaratna WP, Seward JB, Appleton CP, Douglas PS, JK O, Tajik AJ, et al. Left atrial size: physiologic determinants and clinical applications. J Am Coll Cardiol. 2006;47(12):2357-63. https://doi. org/10.1016/j.jacc.2006.02.048.

12. Prioli A, Marino P, Lanzoni L, Zardini P. Increasing degrees of left ventricular filling impairment modulate left atrial function in humans. Am J Cardiol. 1998;82(6):756-61. https://doi.org/10. 1016/S0002-9149(98)00452-4.

13. Takemoto Y, Barnes ME, Seward JB, Lester SJ, Appleton CA, Gersh BJ, et al. Usefulness of left atrial volume in predicting first congestive heart failure in patients $>$ or $=65$ years of age with wellpreserved left ventricular systolic function. Am J Cardiol. 2005;96(6):832-6. https://doi.org/10.1016/j.amjcard.2005.05.031.

14. Fang F, Lee AP, Yu CM. Left atrial function in heart failure with impaired and preserved ejection fraction. Curr Opin Cardiol. 2014;29(5):430-6. https://doi.org/10.1097/HCO.0000000000000091.

15. Melenovsky V, Hwang SJ, Redfield MM, Zakeri R, Lin G, Borlaug BA. Left atrial remodeling and function in advanced heart failure with preserved or reduced ejection fraction. Circ Heart Fail. 2015;8(2): 295-303. https://doi.org/10.1161/CIRCHEARTFAILURE.114. 001667.

16. Patel DA, Lavie CJ, Milani RV, Ventura HO. Left atrial volume index predictive of mortality independent of left ventricular geometry in a large clinical cohort with preserved ejection fraction. Mayo Clin Proc. 2011;86(8):730-7. https://doi.org/10.4065/mcp.2010. 0682.

17. Levy D, Garrison RJ, Savage DD, Kannel WB, Castelli WP. Prognostic implications of echocardiographically determined left ventricular mass in the Framingham Heart Study. N Engl J Med. 1990;322(22):1561-6. https://doi.org/10.1056/ NEJM199005313222203.
18. Ghali JK, Liao Y, Simmons B, Castaner A, Cao G, Cooper RS. The prognostic role of left ventricular hypertrophy in patients with or without coronary artery disease. Ann Intern Med. 1992;117(10): 831-6. https://doi.org/10.7326/0003-4819-117-10-831.

19. Heinzel FR, Hohendanner F, Jin G, Sedej S, Edelmann F. Myocardial hypertrophy and its role in heart failure with preserved ejection fraction. J Appl Physiol (1985). 2015;119(10):1233-42. https://doi.org/10.1152/japplphysiol.00374.2015.

20. Farber NJ, Reddy ST, Doyle M, Rayarao G, Thompson DV, Olson $\mathrm{P}$, et al. Ex vivo cardiovascular magnetic resonance measurements of right and left ventricular mass compared with direct mass measurement in excised hearts after transplantation: a first human SSFP comparison. J Cardiovasc Magn Reson. 2014;16(1):74. https://doi. org/10.1186/s12968-014-0074-0.

21. Bottini PB, Carr AA, Prisant LM, Flickinger FW, Allison JD, Gottdiener JS. Magnetic resonance imaging compared to echocardiography to assess left ventricular mass in the hypertensive patient. Am J Hypertens. 1995;8(3):221-8. https://doi.org/10.1016/08957061(94)00178-E.

22. Mavrogeni S, Katsi V, Vartela V, Noutsias M, MarkousisMavrogenis G, Kolovou G, et al. The emerging role of cardiovascular magnetic resonance in the evaluation of hypertensive heart disease. BMC Cardiovasc Disord. 2017;17(1):132. https://doi.org/ 10.1186/s12872-017-0556-8.

23. Nagueh SF, Appleton CP, Gillebert TC, Marino PN, JK O, Smiseth $\mathrm{OA}$, et al. Recommendations for the evaluation of left ventricular diastolic function by echocardiography. Eur J Echocardiogr. 2009;10(2):165-93.

24. Rathi VK, Doyle M, Yamrozik J, Williams RB, Caruppannan K, Truman C, et al. Routine evaluation of left ventricular diastolic function by cardiovascular magnetic resonance: a practical approach. J Cardiovasc Magn Reson. 2008;10(1):36. https://doi.org/ 10.1186/1532-429X-10-36.

25. Kozerke S, Schwitter J, Pedersen EM, Boesiger P. Aortic and mitral regurgitation: quantification using moving slice velocity mapping. J Magn Reson Imaging. 2001;14(2):106-12. https://doi.org/10.1002/ jmri.1159.

26. Westenberg JJ, Roes SD, Ajmone Marsan N, Binnendijk NM, Doornbos J, Bax JJ, et al. Mitral valve and tricuspid valve blood flow: accurate quantification with $3 \mathrm{D}$ velocity-encoded MR imaging with retrospective valve tracking. Radiology. 2008;249(3):792800. https://doi.org/10.1148/radiol.2492080146.

27. Brandts A, Bertini M, van Dijk EJ, Delgado V, Marsan NA, van der Geest RJ, et al. Left ventricular diastolic function assessment from three-dimensional three-directional velocity-encoded MRI with retrospective valve tracking. J Magn Reson Imaging. 2011;33(2):3129. https://doi.org/10.1002/jmri.22424.

28. Klein AL, Tajik AJ. Doppler assessment of pulmonary venous flow in healthy subjects and in patients with heart disease. J Am Soc Echocardiogr. 1991;4(4):379-92. https://doi.org/10.1016/S08947317(14)80448-3.

29. Kawaji K, Codella NC, Prince MR, Chu CW, Shakoor A, LaBounty TM, et al. Automated segmentation of routine clinical cardiac magnetic resonance imaging for assessment of left ventricular diastolic dysfunction. Circ Cardiovasc Imaging. 2009;2(6): 476-84. https://doi.org/10.1161/CIRCIMAGING.109.879304.

30. Mendoza DD, Codella NC, Wang Y, Prince MR, Sethi S, Manoushagian SJ, et al. Impact of diastolic dysfunction severity on global left ventricular volumetric filling-assessment by automated segmentation of routine cine cardiovascular magnetic resonance. J Cardiovasc Magn Reson. 2010;12(1):46. https://doi.org/ 10.1186/1532-429X-12-46.

31. Edvardsen T, Rosen BD, Pan L, Jerosch-Herold M, Lai S, Hundley WG, et al. Regional diastolic dysfunction in individuals with left ventricular hypertrophy measured by tagged magnetic resonance imaging - the Multi-Ethnic Study of Atherosclerosis (MESA). 
Am Heart J. 2006;151(1):109-14. https://doi.org/10.1016/j.ahj. 2005.02.018.

32. Morton G, Schuster A, Jogiya R, Kutty S, Beerbaum P, Nagel E. Inter-study reproducibility of cardiovascular magnetic resonance myocardial feature tracking. J Cardiovasc Magn Reson. 2012;14(1):43. https://doi.org/10.1186/1532-429X-14-43.

33. Bolger AF, Heiberg E, Karlsson M, Wigstrom L, Engvall J, Sigfridsson A, et al. Transit of blood flow through the human left ventricle mapped by cardiovascular magnetic resonance. J Cardiovasc Magn Reson. 2007;9(5):741-7. https://doi.org/10. 1080/10976640701544530.

34. Mewton N, Liu CY, Croisille P, Bluemke D, Lima JA. Assessment of myocardial fibrosis with cardiovascular magnetic resonance. $\mathrm{J}$ Am Coll Cardiol. 2011;57(8):891-903. https://doi.org/10.1016/j. jacc.2010.11.013.

35. Martos R, Baugh J, Ledwidge M, O'Loughlin C, Conlon C, Patle A, et al. Diastolic heart failure: evidence of increased myocardial collagen turnover linked to diastolic dysfunction. Circulation. 2007;115(7):888-95. https://doi.org/10.1161/CIRCULATIONAHA. 106.638569 .

36. Borbely A, van der Velden J, Papp Z, Bronzwaer JG, Edes I, Stienen GJ, et al. Cardiomyocyte stiffness in diastolic heart failure. Circulation. 2005;111(6):774-81. https://doi.org/10.1161/01.CIR. 0000155257.33485.6D.

37. Aoki T, Fukumoto Y, Sugimura K, Oikawa M, Satoh K, Nakano M, et al. Prognostic impact of myocardial interstitial fibrosis in nonischemic heart failure. Comparison between preserved and reduced ejection fraction heart failure. Circ J. 2011;75(11):2605-13. https:// doi.org/10.1253/circj.CJ-11-0568.

38. Zile MR, Baicu CF, SI J, Stroud RE, Nietert PJ, Bradshaw AD, et al. Myocardial stiffness in patients with heart failure and a preserved ejection fraction: contributions of collagen and titin. Circulation. 2015;131(14):1247-1259. Study addressing whether patients with HFpEF have increased passive myocardial stiffness In patients with $\mathrm{HFpEF}$ and hypertension there were changes in the extracellular matrix fibrillar collagen and cardiomyocyte titin. https://doi.org/10.1161/CIRCULATIONAHA.114.013215.

39. Broadbent DA, Biglands JD, Larghat A, Sourbron SP, Radjenovic A, Greenwood JP, et al. Myocardial blood flow at rest and stress measured with dynamic contrast-enhanced MRI: comparison of a distributed parameter model with a fermi function model. Magn Reson Med. 2013;70(6):1591-7. https://doi.org/10.1002/mrm. 24611.

40. Flett AS, Hayward MP, Ashworth MT, Hansen MS, Taylor AM, Elliott PM, et al. Equilibrium contrast cardiovascular magnetic resonance for the measurement of diffuse myocardial fibrosis: preliminary validation in humans. Circulation. 2010;122(2):138-44. https://doi.org/10.1161/CIRCULATIONAHA.109.930636.

41. Iles L, Pfluger H, Phrommintikul A, Cherayath J, Aksit P, Gupta $\mathrm{SN}$, et al. Evaluation of diffuse myocardial fibrosis in heart failure with cardiac magnetic resonance contrast-enhanced T1 mapping. J Am Coll Cardiol. 2008;52(19):1574-80. https://doi.org/10.1016/j. jacc.2008.06.049.

42. Sado DM, Flett AS, Banypersad SM, White SK, Maestrini V, Quarta G, et al. Cardiovascular magnetic resonance measurement of myocardial extracellular volume in health and disease. Heart. 2012;98(19):1436-41. https://doi.org/10.1136/heartjnl-2012302346.

43. Mascherbauer J, Marzluf BA, Tufaro C, Pfaffenberger S, Graf A, Wexberg P, et al. Cardiac magnetic resonance post-contrast $\mathrm{T} 1$ time is associated with outcome in patients with heart failure and preserved ejection fraction. Circ Cardiovasc Imaging. 2013;6(6): 1056-65. https://doi.org/10.1161/CIRCIMAGING.113.000633.

44. Su MY, Lin LY, Tseng YH, Chang CC, Wu CK, Lin JL, et al. CMRverified diffuse myocardial fibrosis is associated with diastolic dysfunction in HFpEF. JACC Cardiovasc Imaging. 2014;7(10):991-7. https://doi.org/10.1016/j.jcmg.2014.04.022.

45. Sinkus R, Tanter M, Catheline S, Lorenzen J, Kuhl C, Sondermann $\mathrm{E}$, et al. Imaging anisotropic and viscous properties of breast tissue by magnetic resonance-elastography. Magn Reson Med. 2005;53(2):372-87. https://doi.org/10.1002/mrm.20355.

46. Arani A, Arunachalam SP, Chang IC, Baffour F, Rossman PJ, Glaser KJ, et al. Cardiac MR elastography for quantitative assessment of elevated myocardial stiffness in cardiac amyloidosis. J Magn Reson Imaging: JMRI. 2017;46(5):1361-7. https://doi.org/ 10.1002/jmri.25678. This is an important manuscript in cardiac magnetic resonance elastography as it uses classical elastography to define systolic and diastolic pressures for the heart 